\title{
Systematic review in scientific controversy: the example of water fluoridation, an open access review
} D Richards ${ }^{1}$, P Mansfield ${ }^{2}$ and J Kleijnen ${ }^{3}$

${ }^{1}$ Director, Centre for Evidence-based Dentistry, Oxford, UK, ${ }^{2}$ Director of Templegarth Trust, Louth, UK, ${ }^{3} \mathrm{NHS}$ Centre for Reviews and Dissemination, University of York, York, UK

The systematic review of the safety and effectiveness of water fluoridation was an open-access systematic review. This has overcome some of the difficulties encountered in a highly contentious area, but it did not work perfectly. Parties on each side of the controversy were reluctant to abandon their previous positions and endorse the review result whole-heartedly. Surprising progress was made, however, which should encourage greater confidence in future reviews of this kind.

Evidence-Based Dentistry (2002) 3, 32-34. doi:10.1038/sj.ebd.6400121

The systematic review published in $2000^{1,2}$ on the safety and effectiveness of water fluoridation is the first of its type in many respects. We believe that important lessons can be learned from the conduct of this review that can be used to improve the quality of scientific reviews in areas where strong views are held.

Systematic reviews ${ }^{3,4}$ locate, appraise and synthesise evidence from scientific studies in order to provide informative empirical answers to scientific research questions. They are therefore valuable sources of information for decisionmakers. In addition, by identifying what we know and don't know, they are an invaluable first step before carrying out new primary research. Systematic reviews differ from other types of review in that they adhere to a strict scientific design in order to make them more comprehensive, to minimise the chance of bias, and so ensure the views of the authors or being based only on a (possibly biased) selection of the published literature, they contain a comprehensive summary of the available evidence.

Following the decision of the UK Government to conduct a review of the evidence relating to the safety and effectiveness of water fluoridation, the Centre for Reviews and Dissemination (CRD) in York was contracted to carry out the review. One of the early decisions was to appoint an advisory panel to the review. The advisory panel was balanced to represent views from both sides of the fluoridation debate, as well as including those who are neutral. This included doctors, dentists, scientists, consumers, epidemiologists and representatives of the water industry. The review team met with the advisory panel on three occasions. The purpose of the first meeting was to clarify the their reliability. Rather than reflecting protocol, and of the subsequent meetings to discuss progress and drafts of the reports. Members of the advisory panel also had frequent discussions with the review team during the progress of the review.

A website (http://www.york.ac.uk/ inst/crd/fluorid.htm) was established shortly after the first advisory panel meeting. This initially contained the draft protocol and the approved minutes for the advisory meeting. The objective of providing the website was to allow interested groups and individuals open access to the process of the review and give them the opportunity to comment or provide additional information, which could be relevant to the review. A paper detailing the impact of this on the methodology of the review is in preparation.

An important element in the process of the advisory panel meetings was the discussion of the protocol. This discussion focused on identifying the key objectives of the review and the types of evidence required to address these objectives. This resulted in the definition of specific inclusion criteria for each objective and what confounding factors needed to be addressed in the analysis. The review team felt that these discussions were very helpful in preparing the protocol for the review. In addition, the focus on the structured methodology of the systematic review 
was a binding factor in the heterogeneous advisory panel.

These discussions and the open process of the performance of the review 'on the web' convinced the members of the advisory panel that the review team was independent and not working towards predetermined conclusions. In the end, this led to agreement about the protocol and the results of the review. Despite a measure of success, however, there was still a large measure of suspicion and rumour. Some of this could be related to a lack of complete understanding of the systematic review process, which existed on both sides of the argument. Despite the availability of clear information on both the questions being covered by the review and the process of systematic reviewing itself on the website, there was a lack of understanding of the ground rules and the reason for them. This was clearly a deep-seated problem because it was evident in comments from senior academics and professionals as well as members of the public.

The remit from the UK Department of Health was to conduct a systematic review of the evidence for the safety and effectiveness of water fluoridation based on the currently available evidence from population-based studies. This is only a small section of the issues that need to be considered when making the decision of whether or not public water supplies should be fluoridated. By limiting the scope of the review to a manageable area a thorough and high-quality assessment of the available evidence could be conducted. Attempting to review the whole fluoridation issue in one systematic review would be a huge undertaking. In the past other non-systematic reviews ${ }^{5-22}$ have attempted this, which inevitably led to a compromise on quality and accusations of bias, the probity of which cannot be checked.

The open-access format certainly provided an opportunity for a wide range of groups and individuals to submit information, which in some instances amounted to scholarly reviews working from other ground rules than those of the York review. Contributors from both sides of the argument whose comments were not included will understandably be disappointed. Many were rejected because they covered areas which, although relevant to the overall fluoride debate, were outside the focus of the review. Others were rejected because they did not meet the inclusion criteria set at the outset of the review, most usually for lack of baseline information or of an adequate control study.

Drafts of the results of the review appeared on the web before the completion of the report. Although this helped improve both the quality and the readability of the report it has inevitably led to speculation as to its eventual findings. With the results of the report, but not the conclusions, available at an early stage some 'spinning' occurred. ${ }^{23,24}$ The advisory panel and review team have tried hard to ensure that the report is a factual account of the best available evidence on the questions addressed. (Summaries of the evidence related to each of the questions addressed by the report appear later in the journal.)

Although the advisory panel and review team agreed the results of the review, agreement was not reached about the conclusions or, more importantly, the implications of the review. As predicted by the review leader at the first advisory board meeting, this was never going to be a once-and-for-all assessment that would settle the matter about water fluoridation. Systematic reviews are simply not policy decisions. They can only inform such decisions along with many other relevant factors.

This open-access systematic review has overcome some of the difficulties encountered in a highly contentious area, but it did not work perfectly. Parties on each side of the controversy were reluctant to abandon their previous positions and endorse the review result whole-heartedly. Surprising progress was made, however, which should encourage greater confidence in future reviews of this kind. Prerequisites for this are adequate assurance of the independence of the review team well borne out in this instance - and a far greater grasp in advance of the review process and the reasons for it.

Future research can be more efficient and sharply focused as a result of open reviews of this kind. A consensus on future research priorities was one result of this review. The eventual findings of future work are more likely to be debated rationally and achieve wide consensus than if the review had not taken place. This prospect is a strong argument for rapid extension of the open systematic review process into other controversial areas.

\section{Conclusion}

Willingness to work openly through all the evidence in a contentious issue is essential for efficient use of resources in the widest public interest. Water fluoridation was a hard test of any debating process. Open-access, independent, systematic scientific review proved to be a giant leap in the right direction.

1. NHS Centre for Reviews and Dissemination. A Systematic Review of Water Fluoridation. York: NHS Centre for Reviews and Dissemination, University of York; 2000.

2. McDonagh MS, Whiting PF, Wilson PM, Sutton AJ, Chestnutt I, Cooper J, et al. Systematic review of water fluoridation. BMJ 2000; 321:855-859.

3. NHS Centre for Reviews and Dissemination. Undertaking Systematic Reviews of Research on Effectiveness: CRD's Guidance for Carrying Out or Commissioning Reviews. 2nd Edition. York: NHS Centre for Reviews and Dissemination, University of York; 2001.

4. Chalmers I, Altman DG (Eds). Systematic Reviews. London: BMJ; 1995.

5. Ministry of Health, Department of Health for Scotland, Ministry of Housing and Local Government. The Fluoridation of Domestic Water Supplies in North America as a Means of Controlling Dental Caries. Report of the United Kingdom Mission. London: HMSO. 1953

6. World Health Organisation. Report of Expert Committee on Water Fluoridation. Technical Report Series No 146. Geneva: World Health Organisation; 1958.

7. Ministry of Health, Scottish Office, Ministry of Housing and Local Government. The conduct of the fluoridation studies in the United Kingdom and the results achieved after five years. Reports on Public Health and Medical Subjects. No. 105. London: HMSO; 1962. 
8. Department of Health and Social Security, Scottish Office, Welsh Office. The fluoridation studies in the United Kingdom and the results achieved after eleven years. Reports on Public Health and Medical Subjects. No. 122. London: HMSO; 1969.

9. World Health Organisation. Fluorides and human health. World Health Organisation Monograph Series. No. 59. Geneva: World Health Organisation; 1970.

10. Royal College of Physicians. Fluoride, Teeth and Health. Bath: Pitman Medical; 1976.

11. Clemmesen J. The alleged association between artificial fluoridation of water supplies and cancer: a review. Bull World Health Organ 1983; 61:871-883.

12. Lord Jauncey. Opinion of Lord Jauncey in causa Mrs Catherine McColl (AP) against Strathclyde Regional Council. Edinburgh: Court of Session; 1983.

13. Knox E G. Fluoridation of Water and Cancer: a Review of the Epidemiological Evidence. London: HMSO; 1985

14. Ad Hoc Subcommittee on Fluoride of the Committee to Coordinate Environmental Health and Related Programs. Review of Fluoride: Benefits and Risks. Washington DC: Public Health Service, US Department of Health and Human Services; 1991.

15. Murray JJ, Rugg-Gunn AJ, Jenkins GN. Fluorides in Caries Prevention. 3rd Edn. Oxford: Wright; 1991.

16. National Health and Medical Research Council. The Effectiveness of Water Fluoridation. Canberra: Commonwealth of Australia; 1991.

17. Medical Research Council Physiological Medicine and Infections Board. Report of the Working Group on Fluoridation of Drinking Water - Link With Osteoporosis, 17 December 1993. London: Medical Research Council; 1993.

18. New Zealand Public Health Commission. Fluoridation of Water Supplies. Draft Policy Statement, May 1993. Wellington: Public Health Commission; 1993.
19. National Research Council, National Academy of Sciences Committee on Toxicology. Health Effects of Ingested Fluoride. Washington DC: National Academy Press; 1993.

20. Expert Committee on Oral Health Status and Fluoride Use. Fluorides and oral health. WHO Technical Report Series. No. 846. Geneva: World Health Organisation; 1994.

21. Royal Melbourne Institute of Technology - Key Centre for Toxicology and the Monash University Medical School's Centre for Epidemiology and Preventive Medicine. Review of Water Fluoridation and Fluoride Intake from Discretionary Fluoride Supplements; 1999.

22. National Research Council: National Academy of Sciences Committee on Toxicology. Health effects of ingested fluoride. Washington DC: National Academy Press 1993.

23. Anon. Fluoride: the state of the art and science. REPORT: Newsl UK Public Health Assoc Summer 2000; Issue 4:21-22.

24. British Dental Association. British Dental Association News Release 20 Jul 2000

York Fluoride Systematic Review Team Jos Kleijnen, NHS Centre for Reviews and Dissemination, York, UK

Marian McDonagh, NHS Centre for Reviews and Dissemination, York, UK

Kate Misso, NHS Centre for Reviews and Dissemination, York, UK

Penny Whiting, NHS Centre for Reviews and Dissemination, York, UK

Paul Wilson, NHS Centre for Reviews and Dissemination, York, UK

Ivor Chestnutt, Dental Public Health Unit, Cardiff, Wales, UK
Jan Cooper, Dental School, University of Wales College of Medicine, Cardiff,

Wales, UK

Elizabeth Treasure, Dental School, University of Wales College of Medicine, Cardiff, Wales, UK

\section{Systematic Review Advisory Panel}

Chair: Trevor Sheldon, York Health

Policy Group, University of York, York, UK

Earl Baldwin of Bewdley, House of Lords, London, UK

Iain Chalmers, UK Cochrane Centre, Oxford, UK

Sheila Gibson, Glasgow Homeopathic

Hospital, Glasgow, Scotland, UK

Sarah Gorin, Help for Health Trust,

Winchester, UK

Mike Lennon, Chairman of the British Fluoridation Society, Department of Clinical Dental Sciences, University of Liverpool School of Dentistry, Liverpool, UK

Peter Mansfield, Director of Templegarth

Trust, Louth, UK

John Murray, Dean of Dentistry, University of Newcastle, Newcastle upon Tyne, UK

Jerry Read, Department of Health, London, UK

Derek Richards, Centre for EvidenceBased Dentistry, Oxford, UK

George Davey Smith, Department of Social Medicine, University of Bristol, Bristol, UK

Pamela Taylor, Water UK, London, UK 